\title{
(Wissenschafts-)Kultur der Digitalität
}

\author{
A. Lasch \\ Professur für germanistische Linguistik und Sprachgeschichte, Institut für Germanistik, Fakultät SLK, TU Dresden
}

\begin{abstract}
Die Rede von der "Kultur der Digitalität" ([7] Stalder 2019) sorgt in Universitäten regelmäßig für Unruhe. Der Titel löst dann Irritationen aus, wenn damit die Implikation verbunden ist, dass von einem Umbruch, von einer Ablösung auszugehen ist, dessen Folge es sei, dass die Universität, wie wir sie kennen, fortan nicht mehr existiere. Das Gegenteil ist der Fall - denn, wenn man das eine tun kann, bedeutet es nicht zugleich, dass man das andere lassen muss ([1] Lasch 2020). In meinem kurzen Beitrag möchte ich deshalb (1) das Konzept erläutern, es (2) auf unsere Communitas Universität übertragen und (3) ein Projekt vorstellen, das sich konsequenterweise genau dann etablieren kann, wenn man Universität als Universitas in einem umfassenden Sinn als Institution einer "(Wissenschafts-)Kultur der Digitalität" versteht ([2] Lasch \& Schoop 2021): „virTUos" (Virtuelles Lehren und Lernen an der TU Dresden im Open Source-Kontext).

Talking about a "digital culture" ([7] Stalder 2019, also: the digital condition) regularly causes a stir in universities. The phrase causes irritation insofar as it implies that there is an upheaval, a replacement, the consequence of which is that the university as we know it will henceforth cease to exist. The opposite is true - because if you can do one thing, it does not mean at the same time that you have to leave the other ([1] Lasch 2020). In my short contribution, I would therefore like to (1) explain this phrase, (2) apply it to our communitas university, and (3) present a project that can be established precisely when university as universitas is understood in a comprehensive sense as an institution of a "(scientific) digital culture" ([2] Lasch \& Schoop 2021): "virTUos" (Virtual Teaching and Learning at the TU Dresden in an Open Source Context).
\end{abstract}

*Corresponding author: alexander.lasch@tu-dresden.de 


\section{Kultur der Digitalität}

In einer Kultur der Digitalität (nach [7] Stalder 2019) zu leben, bedeutet, dass sich Transformationen und Verwerfungen von Formen des gesellschaftlichen Umgangs mit Wissen nicht erst andeuten, sondern bereits Realität sind. Damit stehen etablierte Prozesse der Wissensaneignung, der Hervorbringung und Kommunikation von Wissen sowie der Status von Wissen selbst und Formen seiner adäquaten Weitergabe zur Disposition. Die Frage, was Wissen sei und wem es gehöre, war noch nie so offen wie heute. Ein Rückzug auf eine vordigitale Kultur jedenfalls ist keine Option. Wenn diese Option also ausscheidet, bleibt nur die Frage, wie man sich zum aktuell sich vollziehenden Transformationsprozess verhält und $o b$ und wie man inn mitgestalten möchte. Ich bin der Auffassung, dass Universitäten als Akteur:innen in diesem Prozess auftreten und sich positionieren müssen.

Stalder spricht von drei zentralen Merkmalen, die eine Kultur der Digitalität prägen, und die alte kulturelle Ordnungen zum Einsturz bringen: Referentialität, Gemeinschaftlichkeit und Algorithmizität. Unsere Gesellschaft und ihre Institutionen, so Stalder, stehen am Scheideweg: „Unser Handeln bestimmt, ob wir in einer postdemokratischen Welt der Überwachung und der Wissensmonopole oder in einer Kultur der Commons und der Partizipation leben werden." ([7] Stalder 2019, Klappentext) Mit Referentialität spricht Stalder an, dass sich Wissen (als Herrschaftswissen) nicht mehr hegemonial besitzen, verschließen, verwalten und verbuchen oder wie ein Schatz hüten lässt, sondern ganz unabhängig (z.B. von Diskursen über Urheberrecht) verfügbar und neu referentialisiert wird. Durch digitale Praktiken wird dieser Prozess, der in Kulturtechniken wie Kopie und Collage längst bekannt und benannt ist, drastisch beschleunigt: „Kulturelle Werke aller Art werden in einem umfassenden, praktischen Sinn frei verfügbar, trotz bestehender rechtlicher und technischer Einschränkungen." ([7] Stalder 2019: 112) Ähnlich katalysatorisch wirken Gemeinschaften im digitalen Raum, die er mit dem Prinzip der Gemeinschaftlichkeit adressiert. Stalder betont, dass neue epistemische communities of practice, in denen Wissen auf eine neue Art, auf alternativen Wegen und
Praxisfeldern prozessiert wird, in Konkurrenz treten zu den etablierten Institutionen und Gemeinschaften der vordigitalen Kultur. „Sie [sc. communities of practice als epistemische Gemeinschaften] entstehen in einem Praxisfeld, geprägt durch informellen, aber strukturierten Austausch, sind fokussiert auf die Generierung neuer Wissens- und Handlungsmöglichkeiten und werden zusammengehalten durch die reflexive Interpretation der eigenen Praxis. Speziell der letzte Punkt [...] macht die zentrale Rolle der gemeinschaftlichen Formation aus." ([7] Stalder 2019: 136). Und Algorithmizität ist Kennzeichnen von Techniken, die uns sowohl vollkommen neue Erschließungs- und Analysemöglichkeiten wie Produktionsweisen eröffnen, uns aber auch vor neue Herausforderungen stellen, die unsere eingeübten kulturellen Praxen relativieren: „Tätigkeiten, die noch vor Kurzem unzweifelhaft der menschlichen Intelligenz vorbehalten schienen, beispielsweise das Verfassen von Texten oder die Inhaltsanalyse von Bildern, übernehmen inzwischen immer häufiger Maschinen." ([7] Stalder 2019: 173)

Besonders der letzte Aspekt sollte uns Ansporn sein und uns immer wieder zu denken darüber geben, dass ein konsequenter Abschluss nach außen und ein Festhalten an etablierten Formen des Wissenstransfers zu einer Verfestigung der Institutionen führen wird, die sich seit Jahrhunderten der Erarbeitung, Bewahrung und Weitergabe von Wissen verschrieben haben, nicht zu ihrer Vitalisierung.

\section{Universitäten in der Wissensgesell- schaft des 21. Jahrhunderts}

Wir sehen uns also, als eine Communitas, deren wichtigstes Merkmal sei, Wissen zu schaffen und zu prozessieren, kulturellen Prozessen ausgesetzt, die unseren institutionalisierten Umgang mit Wissen permanent herausfordern. Eine Universität ist, im Kern, eine (hoch-) mittelalterliche Institution. Auch die jüngeren Universitäten der Bundesrepublik der 1970er Jahre oder die Gründungen und Erweiterungen in den Neuen Ländern nach 1990 haben Ordnungen und dieses Verständnis übernommen. Eine Universität ist eine selbstverwaltete 
Communitas, eine im Wortsinne universelle Gemeinschaft des Forschens, Lehrens und Lernens. Eine Universität schafft hybride und volatile Formen des Wissens: Es ist im Selbstbild immer dies, vorläufig. Interessanterweise korreliert dies in besonderer Weise mit den Prinzipien, die Stalder für eine Kultur der Digitalität ausmacht, in die Universitäten als Institutionen längst gestellt sind. Sie tragen nicht nur die Traditionen ererbter Vergemeinschaftung und bringen neue Communities hervor, von denen Stalder spricht, sondern sie tragen auch die damit einhergehenden Konflikte innen und nach außen aus: „Die alten Ordnungen, in denen kulturelles Material bisher gefiltert, organisiert und zugänglich gemacht wurde [...], können diesen Strom weder im Kleinen noch im Großen kanalisieren. Sie fungieren kaum mehr als Gatekeeper zwischen den Bereichen, die einst mit ihrer Hilfe als, privat' und ,öffentlich' definiert wurden." ([7] Stalder 2019: 114) Welche Chancen eröffnen sich, wenn wir Universitäten wieder als Orte denken, in denen nie nur ein Weg der richtige war? Ausgangsbeobachtung, und diese wurde im letzten Jahr besonders in den Vordergrund gerückt, ist, dass technologieskeptische Debatten verdecken, sich mit der Frage auseinanderzusetzen, was Universität sein kann und will. Dabei geht es um viel mehr (vgl. [1] Lasch 2020: 241-244):

- Partizipation: Universitäten sind Orte des Austauschs, an denen nicht nur gemeinsam gearbeitet, sondern auch Gemeinschaft gemeinsam gestaltet wird.

- Transparenz: Wir legen Wert auf Nachvollziehbarkeit. Wie können Forschungsdaten so generiert werden, dass ihre Genese transparent ist? Wie ist die Interpretation von Forschungsdaten zu gestalten, damit sie nicht hermetisch ist?

- Sichtbarkeit: Wie können Forschungsdaten und -ergebnisse sichtbar gemacht werden? Wie kann man Lehre öffnen? Wie kann man sichtbare Forschungsdaten und Lehr- und Lernmaterial oder Ergebnisse der Lehre freigeben und dennoch schützen?

- Vernetztheit: Wie können Daten und Ergebnisse aus Forschung und Lehre effektiv relationiert werden? Wie kann man dies im akademischen Verständnis verankern?
- Freigebigkeit: Welchen Status haben unsere Daten? Wie kann man von ihnen gemeinsam profitieren? Wie fördert man in der akademischen Bildung die Bereitschaft zu teilen?

- Zusammenarbeit: Welche Forschungsdaten und -ergebnisse und welche Lehr- und Lerninhalte können gemeinsam erarbeitet werden? Wie kann man in Forschung und Lehre zur Kollaboration motivieren?

- Zielorientierung: Welche Zielvorstellungen können nur bzw. effektiver durch kollaboratives Arbeiten erreicht werden? Können neue Zielvorstellungen (für die eigene oder gemeinsame Arbeit) durch Kollaboration entstehen? Wie und wo können Zielstellungen expliziert werden?

- Werteorientierung: Wie können wir in Forschung und Lehre für diese Prinzipien werben?

Die Antworten auf diese Frage laufen alle hier zusammen: Openness. Drei Beispiele möchte ich noch im Detail in diesem Abschnitt vorstellen, die ich als Ausdruck einer Wissenschaftskultur der Digitalität verstehen möchte und die mit je unterschiedlicher Gewichtung den genannten Prinzipien verpflichtet sind. Das eine ist, exemplarisch, das Blog Lingdrafts. Linguistische Werkstattberichte. Das andere sind Open Educational Resources (OER). Das dritte sind kollaborative Projekte mit Studierenden. Wie ich vor der Vorstellung des Projektvorhabens virtTUos (Abschnitt 3) zu zeigen versuche, ist es vor allem das Handlungsfeld der akademischen Hochschullehre, das uns gestalterische Freiheiten eröffnet.

\subsection{Lingdrafts}

Mit Lingdrafts haben wir mit interessierten Wissenschaftler:innen ohne Berücksichtigung von Statusgruppenunterschieden gemeinsam vor zwei Jahren ein Blogprojekt aufgesetzt, um uns an der Schnittstelle zur Öffentlichkeit zu positionieren, unsere Forschungsfragen sichtbar vorzustellen und Anschluss an tagesaktuelle Themen zu suchen. Unser Redaktionsteam ist mittlerweile bunt und groß, kommuniziert via Messenger und vor allem auf Augenhöhe. Es ist ein echtes Gemeinschaftsprojekt, eine dieser 
epistemischen communities of practice, von denen Stalder spricht. Blogkommunikation zeichnet sich durch Visibilität, Aktualität, Reziprozität und Resonanz, Hybridität und Volatilität sowie eine spezifische Medialität aus (vgl. [1] Lasch 2020: 238). Sie läuft damit den etablierten akademischen Vermittlungspraktiken entgegen, folgt zum anderen aber den eben skizzierten Prinzipien der neuen communities. Wir können in einer Wissenschaftskultur der Digitalität also die Formen mitentwickeln und Möglichkeiten ausschöpfen, Wissen auf alternativem Weg zu prozessieren - in der Communitas Universität. Wir sind uns sicher, dass es der richtige Weg ist, neue Impulse in die Diskussionen unserer Universitäten zu tragen, wie es gerade auch mit diesem Beitrag geschieht.

\subsection{Open Educational Resources (OER)}

Teilhabe und Nachnutzung im gemeinsamen Verfolgen von Zielen gelingt vor allem dann gut, wenn man freigebig Inhalte miteinander teilt, ohne Zugewinninteresse. Genau das ist Anliegen von Open Educational Resources (OER). Zwei Herausforderungen sind damit verbunden: Welche Inhalte darf man als OER bereitstellen und wie kann eine Qualitätssicherung realisiert werden? Beide Fragen berühren unsere Institution Universität im Kern: Wie machen wir unser vorläufig erarbeitetes Wissen sicht-, nutz- und kommunikativ anschließbar? Gleiches gilt für Open Access (OA), dem Modus, in welchem dieser Artikel erscheint, und generell Open Educational Practices (OEP). Um die OER-Diskussion an unserer Universität auf einer belastbare Basis zu stellen, wurde mit interessierten Wissenschaftler:innen des Bereichs Geistes- und Sozialwissenschaften (GSW) und der Sächsischen Landesbibliothek - Staatsund Universitätsbibliothek Dresden (SLUB) ein OER-Display realisiert, das nicht nur OER erklärt, sondern auch Best Practices vorstellt. Wichtiges Element ist eine OER-Beratung, die alle Mitarbeiter:innen und auch Studierende in Anspruch nehmen können. Weiterbildungsangebote des Zentrums für interdisziplinäres Lernen und Lehren (ZiLL) flankieren diese Bemühungen.

\subsection{Kollaboratives Arbeiten}

In der Praxis kann das kollaborative Arbeiten mit Studierenden, das dritte Beispiel, neue Potentiale offenlegen. Prof. Dr. Simon Meier-Vieracker vom Lehrstuhl für Angewandte Linguistik am Institut für Germanistik bspw. treibt, wie ich, Formen offener Wissenschaftskultur massiv voran. Er bloggt "fußballlinguistisch", ist auf Twitter, Instagram und TikTok aktiv. Er produziert Vid- und Podcasts, die er offen zugänglich hält. Mit Studierenden entstehen WiKis und Podcasts - wie selbstverständlich, auf Augenhöhe. Viele dieser linguistischen Inhalte sind OER und liegen auf erwähntem Display, andere nicht. Einige besprechen wir auf Lingdrafts, andere nicht. Und wir sind im Moment damit beschäftigt, Sammlungen anzulegen (z.B. unter TelekollegLinguistik), die die Vielfalt der Angebote dokumentieren und die (auch als neue Formen der Wissenschaftskommunikation) Schnittstellen zur Öffentlichkeit etablieren, ohne dass wir dabei den Anspruch vorantragen, dass die Angebote bitte dann auch von Öffentlichkeit(en) wahrgenommen werden dürfen (vgl. zu dieser Erwartungshaltung im Kontext von Blogkommunikation [1] Lasch 2020: 239).

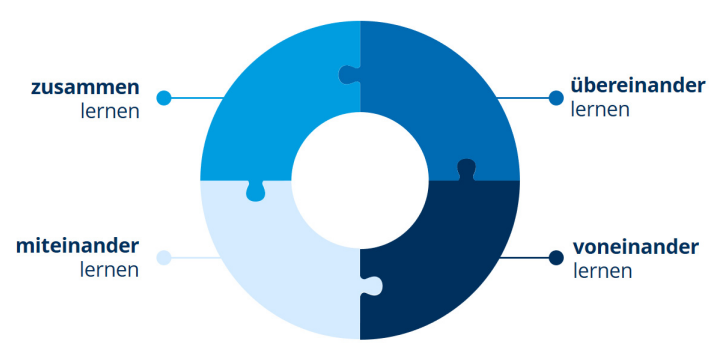

Abb. 1: Lernen in offenen Communitates unter den Vorzeichen der (Wissenschafts-)Kultur der Digitalität. CC BY 4.0 International.

Es sind Experimentierräume, die wir bespielen, um Vorläufiges zu zeigen und auch evaluieren zu lassen: Ich nutze dafür seit mehreren Jahren Formulare, mit denen jederzeit anonymes Feedback durch Studierende ermöglicht wird. Seit dem Wintersemester 2020 habe ich mich dazu entschlossen, dieses Feedback aus Gründen der Transparenz öffentlich zu stellen. Das Feedback fließt direkt in die Formulierung von 
Zielsetzungen ein, in deren Mittelpunkt die Bemühung steht, für die Idee einer offenen Communitas Universität zu begeistern, in der zusammen, übereinander, voneinander und miteinander gelernt wird.

Offenheit ist nicht zu verordnen. Offenheit muss gemeinsam erarbeitet werden. Unsere Hochschulen sahen sich im letzten Jahr unter den Vorzeichen der Pandemie vor massive Herausforderungen gestellt, allen wurde viel und manchmal über die Maßen viel abverlangt. Wir haben viel gelernt und es ist zu hoffen, dass sich manche hochschuldidaktische Entwicklung verstetigt. Für die mittelfristige Verankerung digitaler Prüfungsformate lohnt sich der Einsatz, ebenso wie für die stärkere Verzahnung von Institutionen mit vergleichbaren Anliegen in der Sache (in Dresden sind das die TU Dresden, TUD, die Dresden International University, DIU, die SLUB, und die Carus Akademie am Universitätsklinikum Carl Gustav Carus Dresden, CA/UKD). Digital gestützte Hochschullehre soll in Zukunft immer möglich sein, wo sie didaktisch angezeigt ist. Das kann auch bis hin zu Distanzformaten reichen, um bspw. Blended Learning-Formate curricular zu verankern. So sind internationale Lehr-, Lernund Forschungskooperationen leichter und in vielen Fällen überhaupt erst sinnvoll möglich, sondern es können Barrieren abgesenkt werden, die vorher eine Partizipation am an Universitäten prozessiertem Wissen unnötig erschwerten.

\section{3. virTUos}

Wie ich versucht habe zu zeigen, bringt vor allem die akademische Lehre ideale Bedingungen mit, um mit neuen Formen der Kollaboration, der Dokumentation und Präsentation zu experimentieren. Diese Erfahrungen und die konsequente Weiterentwicklung von digital gestützten Formaten stehen jetzt an der TUD in Kooperation mit der DIU, SLUB und der CA/UKD im inter- und transdisziplinären Lehrund Lernprojekt virTUos (Virtuelles Lehren und Lernen an der TU Dresden im Open SourceKontext) im Fokus, das ich stellvertretend für alle Projektbeteiligten - Henriette Greulich, Simon Meier-Vieracker, Antje Neuhoff, Stefan Odenbach, Ingo Röder, Beatrice Schlegel \& Eric Schoop - vorstellen darf.

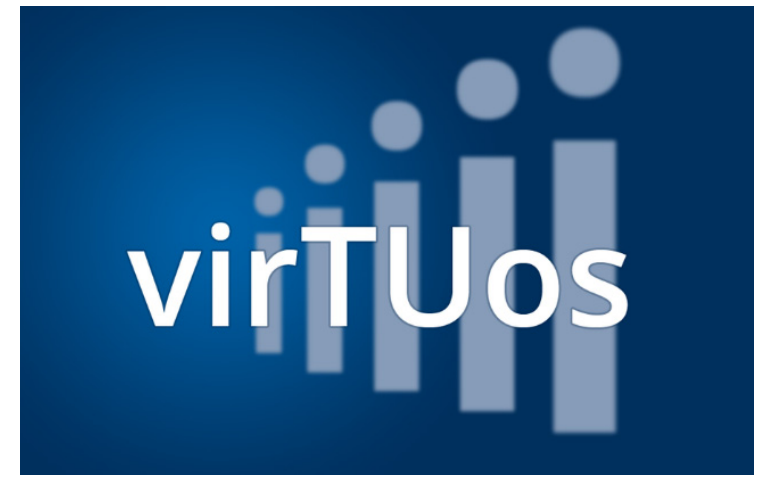

Abb. 2: virTUos. CC BY 4.0 International.

virTUos ist eines von 139 Projektvorhaben, das ab Mitte 2020 von der Stiftung Innovation in der Hochschullehre in der Initiative "Hochschullehre durch Digitalisierung stärken“ über insgesamt drei Jahre gefördert wird, um Veränderungs- und Gestaltungsmöglichkeiten einer digital gestützten Hochschullehre einen institutionellen Rahmen geben zu können.

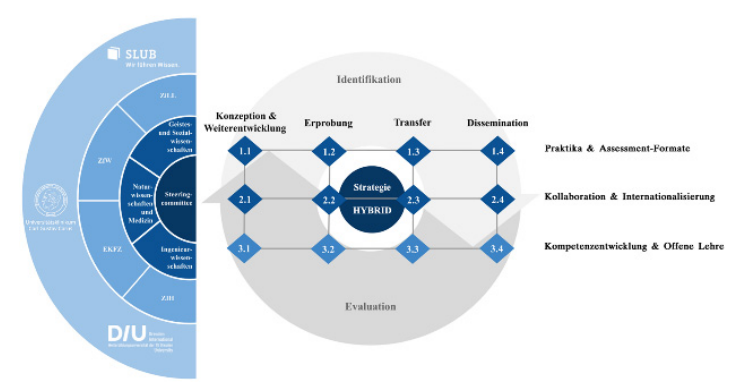

Abb. 3: virTUos-Struktur. links: Orchestriertes Zusammenwirken der virTUos-Fachbereiche, Supportund Transferpartner:innen. rechts: Projektmatrix mit Arbeitspaketen, die sich drei Handlungsfeldern (Zeilen) und vier Projektschritten (Spalten) zuordnen lassen und deren Ergebnisse in einer universitätsweiten Strategie HYBRID integriert und implementiert werden. Eine hochauflösende Variante der Struktur ist hier einzusehen. CC BY 4.0 International.

Denn bisher sind die Ideen und Umsetzungen, wie in Abschnitt 2 an drei Beispielen skizziert, weitgehend insular ohne Verankerung in Curricula und Ordnungen. Dabei kann hybrides Lehren und Lernen eine Säule einer kollaborativen Hochschulentwicklungsstrategie auf den Handlungsfeldern Praktika \& Assessment-Formate (vgl. exemplarisch [3] Pfeiffer u. a. 2018), Kollaboration (exemplarisch [2] Lasch \& Schoop 2021) \& Internationalisierung (exemplarisch [6] Schoop, Clauss \& Safavi 2019) sowie Kompetenzentwicklung (vgl. bspw. [5] Röhle, 
Horneff \& Willemer 2021) \& Offene Lehre (vgl. [4] Redecker \& Punie 2017) sein.

Studierende begleiten und gestalten einen Prozess mit, in dem neuartige Lehr- und Lernszenarien durch interdisziplinäre agile Innovationsteams (vgl. Abb. 4) aus Maschinenwesen, Medizin, Sprachwissenschaft und Wirtschaftswissenschaften mit je konkreten Projektvorhaben getestet, erprobt und ständig weiterentwickelt werden.

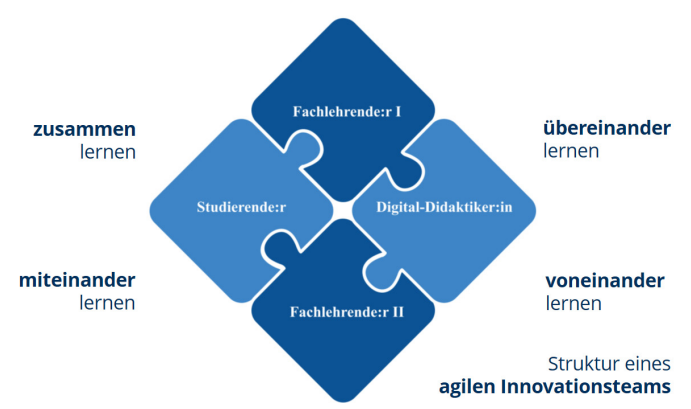

Abb. 4: Struktur eines agilen Innovationsteams. CC BY 4.0 International.

Konzepte Offener Lehre als OEP werden interdisziplinär erarbeitet und hochschulübergreifend weiterentwickelt und genutzt (DIU und CA/UKD); Ergebnisse sind als OER nachnutzbar (SLUB). Ein Integrationsteam koordiniert Skalierung sowie Transfer und entwickelt in der Strategie HYBRID die Verzahnung zentraler und dezentraler Supportstrukturen, zu denen das ZiLL, das Zentrum für Weiterbildung (ZfW), das Zentrum für Informationstechnik und Hochleistungsrechnen (ZIH) sowie das Else KrönerFresenius-Zentrum für Digitale Gesundheit (EKFZ) gehören, entlang der Anforderungen für Studiengangsentwicklung und prüfungsrechtliche Auslegung. Um die innovativen Ideen an der Universität sichtbar zu machen, ist die institutionelle Verankerung und Realisierung der in virTUos erarbeiteten Lösungen in enger Absprache mit der Universitätsleitung erforderlich. So kann ein relevanter Beitrag zur universitätsweiten Harmonisierung digitaler Lehr- und Lern-Ansätze geleistet werden, was gleichzeitig die strukturelle Voraussetzung dafür darstellt, dass eine Kultur digitalen Lernens auf den anvisierten Handlungsfeldern gestärkt und für eine (Wissenschafts-)Kultur der Digitalität geworben wird.

\section{Literatur}

[1] Lasch A (2020): Partizipationswunsch oder Prokrastinationsverdacht? Wissenschaftsvermittlung auf Blogs. In: Marx K, Lobin H \& Schmidt A (Hg.): Deutsch in Sozialen Medien. Interaktiv - multimodal - vielfältig (Jahrbuch des IDS 2019). Berlin, Boston: De Gruyter, 233-245. DOI: https://doi.org/10.1515/9783110679885-012.

[2] Lasch A \& Schoop E (2021): Beispiele des kollaborativen Lernens im virtuellen Raum und Ausblick einer Lernkultur der Digitalität. In: Zukunft Bildung. Fachimpulse der virtuellen Bildungskonferenz 2020 (Microsoft Envision Education 2020). 63-72. Online verfügbar.

[3] Pfeiffer M et al. (2018): IMHOTEP: virtual reality framework for surgical applications. In: IJCARS 13, 741-748. DOI: https://doi.org/10.1007/s11548-0181730-x.

[4] Redecker C \& Punie Y (2017): European Framework for the Digital Competence of Educators (DigCompEdu). Luxembourg. DOI:

https://doi.org/10.2760/159770.

[5] Röhle A, Horneff H \& Willemer MC (2021): Praktische Lehre im Medizinstudium in Zeiten von COVID19. Bericht über die COVID-19-bedingte Umgestaltung der peergestützten Lehre im Skillslab mithilfe eines Inverted Classroom Formats. In: GMS J Med Educ 38(1). DOI: https://doi.org/10.3205/zma001398.

[6] Schoop E, Clauss A \& Safavi A (2020): A Framework to Boost Virtual Exchange through International Vir tual Collaborative Learning: The German-Iranian Example. In: Virtual Exchange Borderless Mobility between the European Higher Education Area and Regions Beyond. Bonn. Stand: 15.02.2021.

[7] Stalder F (2019). Kultur der Digitalität. Berlin: Suhrkamp. 